Pacific Journal of Mathematics

MINIMAL NONPERMUTATIVE PSEUDOVARIETIES OF 


\title{
MINIMAL NON-PERMUTATIVE PSEUDOVARIETIES OF SEMIGROUPS. II
}

JORGE AlMEIDA

\begin{abstract}
This paper is the continuation of a previous paper in which all minimal non- $\mathscr{P}$-permutative pseudovarieties of $\mathscr{C}$ were determined where $\mathscr{P}$-permutativity was one of several conditions implying permutativity and $\mathscr{C}$ was the class of either (finite) groups, monoids or semigroups. In this work, the most general case of this type is treated, namely when $\mathscr{P}$-permutativity is permutativity and $\mathscr{C}$ is the class of all finite semigroups.
\end{abstract}

The notations and conventions adopted in this paper were introduced in [1].

1. Introduction. This paper is the continuation of [1] and uses the notation and conventions introduced there. In [1] we determined all minimal non- $\mathscr{P}$-permutative pseudovarieties of $\mathscr{C}$ where $\mathscr{P}$-permutativity was one of several conditions implying permutativity and $\mathscr{C}$ was the class of either (finite) groups, monoids or semigroups. Here, we treat the most general case of this type, namely when $\mathscr{P}$-permutativity is permutativity and $\mathscr{C}$ is the class of all finite semigroups.

2. Some non-permutative semigroups. In this section, we introduce some semigroups which will play an important role in the sequel. We also indicate a finite basis of identities for each of them.

Recall that any completely simple semigroup is isomorphic to a Rees $I \times \Lambda$ matrix semigroup $\mathscr{M}(G ; I, \Lambda ; P)$ over a group $G$ with sandwich matrix $P=\left(p_{\lambda i}\right)$ (see Clifford and Preston [3]). For a prime number $p$, we let

$$
K_{p}=\mathscr{M}\left(\mathbf{Z}_{p} ; 2,2 ;\left[\begin{array}{ll}
0 & 0 \\
0 & 1
\end{array}\right]\right) .
$$

Rasin [5] has given a complete description of the lattice of varieties of completely simple semigroups over abelian groups. The appropriate universal algebraic type to deal with arbitrary completely simple semigroups involves not only one binary operation (product), but also one unary operation (inversion within the group containing a given element). However, in the context of finite semigroups, the need for the unary operation disappears, since the (group) inverse of an element is then one of its (positive) powers. 
A regular semigroup $S$ is said to be orthodox if the set $E(S)$ of its idempotents is a subsemigroup of $S . S$ is said to be an orthogroup if it is an orthodox union of groups (cf. Clifford [2]).

From the results of Rasin [5], one easily deduces that if $S$ is a finite non-orthodox completely simple semigroup over an abelian group, then $K_{p} \in \underline{V}(S)$ for some prime $p$. Moreover, the $\underline{V}\left(K_{p}\right)$ ( $p$ prime) are minimal non-permutative pseudovarieties of semigroups; they are defined by the following identities:

$$
\underline{V}\left(K_{p}\right)=\llbracket x^{p+1}=x,(x y x)^{p}=x^{p}, x y x z x=x z x y x \rrbracket
$$

and $K_{p}$ is a generator of minimum size of this pseudovariety.

Let

$$
Y=\left\langle e, s, f ; e^{2}=e, f^{2}=f, e s=s=s f, e f=f e=0\right\rangle .
$$

Then $Y=\{e, s, f, 0\}$ and, from Edmunds [4], we obtain

$$
\underline{V}(Y)=\llbracket x^{2}=x^{3}, x y x=x^{2} y^{2}=y^{2} x^{2} \rrbracket ;
$$

we also observe that $Y$ is a generator of minimum size of $\underline{V}(Y)$.

The last special semigroup we need to consider is the following:

$$
Q=\left\langle e, s, t ; e^{2}=e, e s=s, t=t e, s e=e t=t s=0\right\rangle .
$$

We note that $Q=\{e, s, t, s t, 0\}$.

A characterization of the identities which fail in $Q$ will be crucial later on. To describe it, we require some further terminology. We let $X=$ $\left\{x_{1}, x_{2}, \ldots, y_{1}, y_{2}, \ldots, x, y, z, t, \ldots\right\}$ be a countable set of variables; let $X^{+}$be the free semigroup on $x$ and let $X^{*}=X^{+} \cup\{1\}$, where 1 denotes the empty word. For a word $w \in X^{+}, c(w)$ denotes the set of all variables which occur in $w$, and $|w|_{x}$ denotes the number of occurrences of the variable $x$ in $w$. Two identities are said to be equivalent if they hold in exactly the same semigroups. $S \vDash u=v$ means that the identity $u=v$ holds in the semigroup $S$. $\Sigma \vdash u=v$ means that $u=v$ is a consequence of the set $\Sigma$ of identities.

LeMma 2.1. An identity $u=v$ fails in $Q$ if and only if it satisfies (up to equivalence) one of the following conditions:

(i) $c(u) \neq c(v)$;

(ii) there exists $x \in X$ such that $|u|_{x}=1$ and $|v|_{x}>1$;

(iii) there exist $y, z \in X$ such that $u=u_{1} y z u_{2}$ with $|u|_{y}=|u|_{z}=1$ and some $u_{1}, u_{2} \in X^{*}$ while, either $v=v_{1} z v_{2} y v_{3}$ for some $v_{1}, v_{2}, v_{3} \in X^{*}$, or $v=v_{1} y v_{2}^{\prime} z v_{3}$ for some $v_{1}, v_{3} \in X^{*}$ and some $v_{2}^{\prime} \in X^{+}$; 
(iv) there exists $y \in X$ such that $|u|_{y}=1$ and every variable which occurs to the right (left) of $y$ in $u$ occurs only once in $u$, while in $v$ there is some variable which occurs repeatedly and at least once to the right (left) of $y$.

Proof. If (i) holds, then no nontrivial semilattice satisfies $u=v$; since the subsemigroup $\{e, 0\}$ of $Q$ is a semilattice, we obtain $Q \not \neq u=v$.

If (ii) holds, substitute st for $x, e$ for every other variable in $u=v$. This yields the value st and $u$ but 0 for $v$. Hence, $Q \not \neq u=v$.

If case (iii) holds, we substitute $s$ for $y, t$ for $z$ and $e$ for every other variable in $u=v$ to obtain the value st for $u$ and 0 for $v$. Hence, $Q \not \forall u=v$.

Suppose condition (iv) holds, say with the "right" option. Let $y$ be the rightmost variable satisfying (iv). By the above, we may assume that (i)-(iii) fail. Then, $u=u^{\prime} y$ for some $u^{\prime} \in X^{+}$. We substitute $s$ for $y$ and $e$ for every other variable in $u=v$ to obtain the values $s=e s$ for $u$ and $s e=0$ for $v$. Hence $Q \not \not u=v$.

The above establishes half of the lemma. For the converse, let $\neg$ denote negation and assume that, up to equivalence, $u=v$ satisfies the conjunction $(C)$ of conditions $\neg$ (i) $-\neg$ (iv). We show that then $Q \vDash u=v$. Let

$$
\begin{array}{r}
\Sigma=\left\{x^{2}=x^{3}, x y^{2} x=x^{2} y^{2}=y^{2} x^{2}, x^{2} y z^{2}=z^{2} y x^{2}, x y x=x^{2} y x^{2},\right. \\
x y x z x=x z x y x\} .
\end{array}
$$

Simple direct calculations show that $Q \vDash \Sigma$. Thus, it suffices to show $\Sigma \vdash u=v$.

We note that, since each element of $\Sigma$ satisfies $(C)$, if the identities $u=v$ and $u^{\prime}=v^{\prime}$ are equivalent in the presence of $\Sigma$, then $u^{\prime}=v^{\prime}$ satisfies $(C)$ if and only if $u=v$ does. Moreover, using $\Sigma$, any word $w$ can be reduced to one of the form

$$
w^{\prime}=w_{0} x_{1}^{2} w_{1} x_{2}^{2} w_{2} \cdots x_{n-1}^{2} w_{n-1} x_{n}^{2} x_{n+1}^{2} \cdots x_{m}^{2} w_{n}
$$

where $n, m \geq 0, x_{i}(i=1, \ldots, m)$ are not necessarily distinct variables, $c\left(w_{i}\right) \cap c\left(w_{j}\right)=\varnothing$ for $i \neq j, \quad x_{i} \notin c\left(w_{j}\right) \quad(i=1, \ldots, m ; j=0, \ldots, n)$, $\left|w_{i}\right|_{x} \leq 1$ for every $x \in X, w_{i} \in X^{+}(i=1, \ldots, n-1)$, and $w_{0}, w_{n} \in X^{*}$; up to the relative order of the $x_{i}^{2}$, the relative order of the $w_{i}(i=1, \ldots$, $n-1$ ), and the positive number of their occurrences, this form is unique.

Let $u^{\prime}, v^{\prime}$ be words in the previous canonical form obtained respectively from $u, v$ using $\Sigma$, say

$$
u^{\prime}=u_{0} x_{1}^{2} u_{1} x_{2}^{2} u_{2} \cdots x_{n-1}^{2} u_{n-1} x_{n}^{2} x_{n+1}^{2} \cdots x_{m}^{2} u_{n}
$$


and

$$
v^{\prime}=v_{0} y_{1}^{2} v_{1} y_{2}^{2} v_{2} \cdots y_{k-1}^{2} v_{k-1} y_{k}^{2} y_{k+1}^{2} \cdots y_{l}^{2} v_{k} .
$$

By a previous remark, $u^{\prime}=v^{\prime}$ satisfies $(C)$. By $\neg$ (i) and $\neg$ (ii), we may assume that $m=l$ and $x_{i}=y_{i}(i=1, \ldots, m)$. By $\neg$ (ii) and $\neg$ (iii), the variables in $c\left(u_{0} u_{1} \cdots u_{n}\right)=c\left(v_{0} v_{1} \cdots v_{k}\right)$ appear in the same order in $u^{\prime}$ as in $v^{\prime}$, and appear in adjacent positions on one side of $u^{\prime}=v^{\prime}$ if and only if they do so on the other side. Finally, by $\neg$ (iv), $u_{0}=1$ if and only if $v_{0}=1$, and $u_{n}=1$ if and only if $v_{k}=1$. Hence $k=n$ and $u_{i}=v_{i}$, $(i=0,1, \ldots, n)$, up to a rearrangement of the $u_{i}(i=1, \ldots, n-1)$. Thus, the words $u^{\prime}$ and $v^{\prime}$ coincide. Therefore $\Sigma \vdash u=v$, as claimed.

As a corollary to the proof of Lemma 2.1, we have the following.

Proposition 2.2.

$$
\begin{aligned}
\underline{V}(Q)=\llbracket x^{2}=x^{3}, x y^{2} x=x^{2} y^{2}=y^{2} x^{2}, x^{2} y z^{2}=z^{2} y x^{2}, x y x & =x^{2} y x^{2}, \\
x y x z x & =x z x y x \rrbracket .
\end{aligned}
$$

REMARK 2.3. Examining the list of non-permutative varieties generated by semigroups of order four given by Edmunds [4], and noting that a semigroup of order less than four is either permutative or idempotent, one can show that $Q$ is a generator of minimum size of $\underline{V}(Q)$.

3. Regular case. Recall from [1] the semigroups $G_{p}, H_{p, q^{\prime}}, N^{1}$, $B(1,2)^{1}$ and $B(2,1)^{1}$. For a semigroup $S, \rho(S)$ denotes the set of regular elements of $S$.

THEOREM 3.1. Let $\underline{V}$ be a pseudovariety of semigroups such that

$$
\begin{aligned}
& G_{p}, H_{p, q} \notin \underline{V} \quad(p, q \text { distinct primes }) \\
& N^{1}, B(1,2)^{1}, B(2,1)^{1} \notin \underline{V} \\
& K_{p} \notin \underline{V} \quad(p \text { prime }) \\
& Y \notin \underline{V}
\end{aligned}
$$

and let $S \in \underline{V}$. Then $\rho(S)$ is a permutative orthogroup.

We prove Theorem 3.1 in a number of steps. Henceforth, $\underline{V}$ and $S$ are as in Theorem 3.1. First of all, because of (1) and (2), it follows from Theorem 5.3 [1] that all monoids in $\underline{V}$ are commutative.

LemMa 3.2. $E=E(S)$ is a subsemigroup of $S$. 
Proof. Let $e, f \in E$ and suppose that $e f \notin E$. We let $S^{\prime}$ denote the subsemigroup of $S$ generated by $\{e, f\}$.

Suppose $e f, f e$ are not $\mathscr{J}$-equivalent in $S^{\prime}$. If $f e \in E$, then $f e=f e f e$ $<_{\mathscr{J}}$ ef. So, we may assume ef ${ }_{\mathscr{J}} f e$. Let $I=\left\{u \in S^{\prime}\right.$ : ef $\left.\nless_{\mathscr{J}} u\right\}$, an ideal of $S^{\prime}$. Then $S^{\prime} / I$ consists of the four elements $e, f$, ef and $0=f e$ (i.e., $S^{\prime} / I$ is isomorphic to the semigroup $D$ of example 3.11 [1]). However, it is easy to check that $S^{\prime} / I \times S^{\prime} / I$ has a subsemigroup isomorphic to $Y$, whence $Y \in \underline{V}$, contradicting (4).

Hence, we have ef $\mathscr{J} f e$ in $S^{\prime}$. Since ef $\mathscr{R} f e$ implies ef $=f e f$ and so ef $\in E$, we deduce that $e f, f e$ are not $\mathscr{R}$-equivalent. Hence, in the $\mathscr{D}$-class of ef in $S^{\prime}, \mathscr{R} \neq \mathscr{H}$. Let ef $\mathscr{R}$ efu but ef $\mathscr{H}$ efu. Since $e, f$ are idempotents, it follows that $e f=(e f)^{k}$ for some $k>1$. Hence, $G=H_{e f}$ is a subgroup of $S^{\prime}$. Moreover, from $e f=(e f)^{k}$ we obtain $f e f e=(f e)^{k+1}$, and so $H_{f e}$ is also a subgroup of $S^{\prime}$. In fact, $R=J_{e f}$ is a completely simple subsemigroup of $S^{\prime}$. Since $(e f)^{k-1}(f e)^{k-1}=(e f e)^{2 k-3} \notin E, R$ is not orthodox. By the results of Rasin quoted in $\S 2$, it follows that $K_{p} \in \underline{V}(R) \subseteq \underline{V}$ for some prime $p$, contradicting (3). This completes the proof of the lemma.

Let $B A_{2}$ denote the Brandt semigroup $\mathscr{M}^{\circ}(\{1\} ; 2,2 ; \Delta)$ (cf. Clifford and Preston [3]). One can easily check that $Y$ is isomorphic to a subsemigroup of $B A_{2} \times B A_{2}$, and that if a semigroup $U$ has a regular $\mathscr{D}$-class which is not a subsemigroup of $U$, then $B A_{2} \in \underline{V}(U)$.

LEMMA 3.3. $\rho(S)$ is a subsemigroup of $S$ and a union of abelian groups.

Proof. By the remarks preceding the lemma, every regular $\mathscr{D}$-class of $S$ is a completely simple subsemigroup of $S$. Hence, $\rho(S)$ is a union of abelian groups.

Let $a, b \in \rho(S)$. Let $e \in E \cap H_{a}, f \in E \cap H_{b}$. By Lemma 3.2, $e f \in E$. Further, if $a^{\prime} a=e$ and $b b^{\prime}=f$, then $a^{\prime}(a b) b^{\prime}=e f$ and $a b=a e f b$. Hence, $a b \mathscr{J}$ ef and so $a b \in \rho(S)$, as desired.

LeMma 3.4. (Yamada [6], p. 375.) $A$ band is normal if and only if it satisfies the identity $x y x z x=x z x y x$, i.e., if and only if all its submonoids are commutative.

COROllaRY 3.5. E is a normal band.

LEMMA 3.6. If $e, f \in E$ and $s \in \rho(S)$, then esef $=e s f$. 
Proof. Let $g \in E \cap H_{e s}$. Then

$$
\begin{aligned}
\text { esef }=\text { esggef } & =\text { esgegf } \quad \text { by normality of } E \\
& =\text { esggf } \quad \text { since } g \mathscr{R} \text { es } \\
& =\text { esf. }
\end{aligned}
$$

LEMMA 3.7. If $e \in E$ and $s, t \in \rho(S)$, then este $=$ esete.

Proof. Let $g \in E \cap H_{e s}, h \in E \cap H_{t e}$. Then

$$
\begin{aligned}
\text { este } & =\text { esgeghte since } e g=g \\
& =\text { esggehte by normality } \\
& =\text { esete. }
\end{aligned}
$$

To complete the proof of Theorem 3.1, we only need to quote Theorem 3.9(iv) [1].

REMARK 3.8. The reader should consult Yamada [6] for further results on regular permutative semigroups.

4. Main result. We are now ready to establish our main theorem.

THEOREM 4.1. A pseudovariety of semigroups is permutative if and only if it does not contain any of the following semigroups:

$$
G_{p}, H_{p, q}, N^{1}, B(1,2)^{1}, B(2,1)^{1}, K_{p}, Y, Q
$$

( $p, q$ distinct primes).

Moreover, the pseudovarieties generated by each of these semigroups are minimal non-permutative and admit the corresponding semigroup in (5) as a generator of minimum size.

To prove Theorem 4.1, all we really need to show is that if $\underline{V}$ is a pseudovariety which does not contain any of the semigroups in (5), then $\underline{V}$ is permutative. We establish this in several steps. By Theorem 5.3 [1], all monoids in $\underline{V}$ are commutative. Henceforth, $S$ denotes a given element of $V$ and we may assume that $\underline{V}(S)=\underline{V}$. We suppose $S$ is not permutative. Also, let $E=E(S)$.

LEMMA 4.2. If $S \not x^{\omega} y z x^{\omega}=x^{\omega} y x^{\omega} z x^{\omega}$, then $S \vDash x^{\omega} y x^{\omega}=$ $\left(x^{\omega} y x^{\omega}\right)^{k+1}$ for some $k>0$.

Proof. Since $Q \notin \underline{V}$, there exists an identity $u=v$ such that $S \vDash u=v$ while $Q \not \forall u=v$. Hence, $u=v$ satisfies one of the conditions (i)-(iv) of Lemma 2.1. 
If (i) holds, then $\underline{V}$ does not contain any non-trivial semilattices. Since $\rho(S)$ is a union of groups subsemigroup of $S$ and $S$ is finite, it follows that $S$ is a nilpotent extension of a completely simple orthodox union of abelian groups. In particular, $S$ is permutative, contradicting our initial assumption.

If (ii) holds, say the variable $y$ satisfies (ii), then substitute $x^{\omega}$ for every variable in $u=v$ other than $y$ and pre- and post-multiply by $x^{\omega}$ to obtain a pseudo-identity of the form $x^{\omega} y x^{\omega}=x^{\omega} y^{k_{1}} x^{\omega} y^{k_{2}} \cdots x^{\omega} y^{k_{r}} x^{\omega}$ with $\sum_{l=1}^{r} k_{l}>1$ which holds in $S$. Then, substituting $x^{\omega} y x^{\omega}$ for $y$, we obtain

$$
S \vDash x^{\omega} y x^{\omega}=\left(x^{\omega} y x^{\omega}\right)^{k+1} \text { for some } k>0 .
$$

Suppose that (iii) holds. Substitute $x^{\omega}$ for every variable in $u=v$ other than $y, z$ to obtain a pseudo-identity $x^{\omega} y z x^{\omega}=x^{\omega} z y x^{\omega}$ or $x^{\omega} y z x^{\omega}$ $=x^{\omega} y x^{\omega} z x^{\omega}$ which holds in $S$, contradicting the hypothesis of the lemma.

Finally, suppose that (iv) holds, say $y$ is the rightmost variable in $u$ satisfying the "right" option of (iv) and suppose that (i), (ii) and (iii) fail. Upon substitution of $x^{\omega}$ for every variable in $u$ other than $y$, we obtain $S \vDash x^{\omega} y=x^{\omega} y x^{\omega}$, again in contradiction with the assumption of the lemma.

LEMMA 4.3. If $e \in E$ and $s, t \in S$, then este $=$ esete.

Proof. Suppose that este $\neq$ esete. Then, by Lemma 4.2, we have

$$
S \vDash x^{\omega} y x^{\omega}=\left(x^{\omega} y x^{\omega}\right)^{k+1} \text { for some } k \geq 2 .
$$

We claim that this leads to a contradiction. We may assume that $\{e, s, t\}$ generates $S$ and that $s=e s$ and $t=t e$.

By (6), every element of $S$ which lies in a submonoid of $S$ also lies in a subgroup of $S$. In particular, $s e=(s e)^{k+1}=s^{k+1} e$, and so se $\mathscr{R} s^{2}$. Similarly, $t^{2} \mathscr{L}$ et.

Since $s t=(s t)^{k+1}=s t s t \cdots s t$, we have $s t \mathscr{J}(t s)^{2}$ and so tst $\mathscr{L}$ st $\mathscr{R} s t s$. From Theorem 3.1, we deduce that $s t s^{2} t \mathscr{J} s t$, whence $s^{2} t \mathscr{J} s t \mathscr{J}$ stse, and so $s t \mathscr{J} s t s e \cdot t s t$, so that $s t \leq_{\mathscr{J}}$ set.

On the other hand,

$$
\begin{aligned}
\text { set } & =(s e t)^{k+1} \quad \text { by }(6) \\
& =s e t^{k} e s^{k} e t \quad \text { since } e S e \text { is commutative } \\
& =s e s^{k} t^{k} e t \quad \text { by Theorem } 3.1, \text { since } s e, s^{k}, t^{k}, e t \in \rho(S) \\
& =s t t^{k-1} s^{k} e t=s e t^{k} s^{k-1} s t \quad \text { since } e S e \text { is commutative. }
\end{aligned}
$$


Hence, set $\mathscr{H}$ st, so that, by (6), $(s t)^{k}=(s e t)^{k}$. Thus,

$$
\begin{aligned}
\text { este } & =s t=s t(s t)^{k}=s t(\text { set })^{k} \\
& =e s t e(s e)^{k-1}(e t)^{k-1} \text { esete }=e(s e)^{k-1} s t(e t)^{k-1} \text { set }=e s^{k} t^{k} \text { sete } \\
& \text { since eSe is commutative } \\
& =e s^{k} \text { sett }^{k} e \quad \text { by Theorem } 3.1, \text { since } s^{k}, t^{k}, \text { set } \in \rho(s) \\
& =e s^{k+1} e t^{k+1} e=(e s e)^{k+1}(e t e)^{k+1}=e \text { esete by (6). }
\end{aligned}
$$

This contradicts the initial assumption in this proof, and thus the lemma is established.

LEMMA 4.4. If $e, f \in E$ and $s \in S$, then esf $=$ esef.

Proof. Suppose that esf $\neq$ esef. We will then reach a contradiction. Here, we may assume that $S$ is generated by $\{e, f, s\}$ and that $s=e s$.

Using Lemma 4.3, we have sef $=$ esefef $=e s f e f=s f e f$. Since $s e f \neq s f$, we get $s f \neq s f e f$ so that we may replace $s$ by $s f$ and still have $e(s f) f \neq$ $e(s f) e f$. Hence, we may assume that $s=s f$. By Theorem 3.1, we may also assume that $s$ is not regular, and so $s<_{\mathscr{J}} e, f$.

Let $I=\{x \in S: x \nless g x\}$. Note that $I$ is an ideal of $S$.

Suppose $s \leq_{\not} e f$. Then, there exist $u, v \in S$ such that $s=e s f=$ euefvf. If $u \nless{ }_{g} s$, then

$$
\begin{aligned}
s & =\text { esf }=\text { efesf } \\
& =\text { efesef by Lemma } 4.3 \\
& =\text { esefef since } e S e \text { is commutative } \\
& =\text { esef, a contradiction. }
\end{aligned}
$$

Hence $u \leq_{\mathscr{J}} s$. It follows by Lemma 4.3 that $s \leq_{\mathscr{J}}$ sef and so $s \mathscr{J}$ sef. Therefore, $s \mathscr{R}$ sef, so that there exists $w \in S$ such that $s=\operatorname{sefwf}$. If $w \leq_{\mathscr{J}} s$, then $s$ is regular, contradicting a previous assumption. Else, $s=s e f e f=s e f$, again a contradiction. Hence, ef $\in I$, whence sef $\in I$ and we may assume $I=\{0\}$.

If $s \leq_{\mathscr{J}} f e$, then there exist $u, v \in S$ such that $s=e s=e u f e v=$ euefev, the last step because of Lemma 4.3. Since $e f=0$, we conclude that $s=0$, contradicting the definition of $I$. Hence, $f e=0$.

Finally, $s e=s f e=0=f e s=f s$. Hence, $S \simeq Y$ and $Y \in \underline{V}$, a contradiction. This completes the proof of Lemma 4.4. 
To finish the proof of Theorem 4.1, we just need to notice that, by Theorem 5.3 [1] and Lemmas 4.3 and 4.4, the conditions of Theorem 3.9(iv) [1] are satisfied for any $S \in \underline{V}$. Hence, $\underline{V}$ is permutative.

\section{REFERENCES}

[1] J. Almeida, Minimal non-permutative pseudovarieties of semigroups I, Pacific J. Math., 121 (1986), 257-270.

[2] A. H. Clifford, The structure of orthodox unions of groups, Semigroup Forum, 3 (1972), 283-337.

[3] A. H. Clifford and G. B. Preston, The Algebraic Theory of Semigroups, Amer. Math. Soc., Math. Surveys, No. 7, Vol. I, Providence, R. I., 1961.

[4] C. C. Edmunds, Varieties generated by semigroups of order four, Semigroup Forum, 21 (1980), 67-81.

[5] V. V. Rasin, On the lattice of varieties of completely simple semigroups, Semigroup Forum, 17 (1979), 113-122.

[6] M. Yamada, Regular semigroups whose idempotents satisfy permutation identities, Pacific J. Math., 21, No. 2 (1967), 371-392.

Received April 10, 1984. This work was supported in part by NSERC Grant A4044.

Centro de Matemática

UNIVERSIDADE DO MINHO

4700 Braga, Portugal 



\title{
PACIFIC JOURNAL OF MATHEMATICS EDITORS
}

V. S. VARADARAJAN (Managing Editor)
University of California
Los Angeles, CA 90024
HEBERT ClEMENS
University of Utah
Salt Lake City, UT 84112
CHARLES R. DEPRIMA
California Institute of Technology
Pasadena, CA 91125

R. FINN

Stanford University

Stanford, CA 94305

HERMANN FLASCHKA

University of Arizona

Tucson, AZ 85721

Ramesh A. Gangolli

University of Washington

Seattle, WA 98195

ROBION KIRBY

University of California

Berkeley, CA 94720
C. C. MOORE

University of California

Berkeley, CA 94720

H. SAMELSON

Stanford University

Stanford, CA 94305

HaRold Stark

University of California, San Diego

La Jolla, CA 92093

\section{ASSOCIATE EDITORS}

\author{
R. ARENS \\ E. F. BECKENBACH \\ B. H. NeumanN \\ F. Wolf \\ K. YoSHIDA \\ (1906-1982)
}

\section{SUPPORTING INSTITUTIONS}

UNIVERSITY OF ARIZONA
UNIVERSITY OF BRITISH COLUMBIA
CALIFORNIA INSTITUTE OF TECHNOLOGY
UNIVERSITY OF CALIFORNIA
MONTANA STATE UNIVERSITY
UNIVERSITY OF NEVADA, RENO
NEW MEXICO STATE UNIVERSITY
OREGON STATE UNIVERSITY

UNIVERSITY OF OREGON

UNIVERSITY OF SOUTHERN CALIFORNIA

STANFORD UNIVERSITY

UNIVERSITY OF HAWAII

UNIVERSITY OF TOKYO

UNIVERSITY OF UTAH

WASHINGTON STATE UNIVERSITY UNIVERSITY OF WASHINGTON

The Supporting Institutions listed above contribute to the cost of publication of this Journal, but they are not owners or publishers and have no responsibility for its content or policies.

Mathematical papers intended for publication in the Pacific Journal of Mathematics should be in typed form or offset-reproduced (not dittoed), double spaced with large margins. Please do not use built up fractions in the text of the manuscript. However, you may use them in the displayed equations. Underline Greek letters in red, German in green, and script in blue. The first paragraph must be capable of being used separately as a synopsis of the entire paper. In particular it should contain no bibliographic references. Please propose a heading for the odd numbered pages of less than 35 characters. Manuscripts, in triplicate, may be sent to any one of the editors. Please classify according to the scheme of Math. Reviews, Index to Vol. 39. Supply name and address of author to whom proofs should be sent. All other communications should be addressed to the managing editor, or Elaine Barth, University of California, Los Angeles, California 90024.

There are page-charges associated with articles appearing in the Pacific Journal of Mathematics. These charges are expected to be paid by the author's University, Government Agency or Company. If the author or authors do not have access to such Institutional support these charges are waived. Single authors will receive 50 free reprints; joint authors will receive a total of 100 free reprints. Additional copies may be obtained at cost in multiples of 50 .

The Pacific Journal of Mathematics is issued monthly as of January 1966. Regular subscription rate: $\$ 190.00$ a year (5 Vols., 10 issues). Special rate: $\$ 95.00$ a year to individual members of supporting institutions.

Subscriptions, orders for numbers issued in the last three calendar years, and changes of address should be sent to Pacific Journal of Mathematics, P.O. Box 969, Carmel Valley, CA 93924, U.S.A. Old back numbers obtainable from Kraus Periodicals Co., Route 100, Millwood, NY 10546.

The Pacific Journal of Mathematics at P.O. Box 969, Carmel Valley, CA 93924 (ISSN 0030-8730) publishes 5 volumes per year. Application to mail at Second-class postage rates is pending at Carmel Valley, California, and additional mailing offices. Postmaster: Send address changes to Pacific Journal of Mathematics, P.O. Box 969, Carmel Valley, CA 93924.

\section{PUBLISHED BY PACIFIC JOURNAL OF MATHEMATICS, A NON-PROFIT CORPORATION}

Copyright $\subset 1986$ by Pacific Journal of Mathematics 


\section{Pacific Journal of Mathematics}

\section{Vol. 121, No. 2 December, 1986}

Jorge Almeida, Minimal nonpermutative pseudovarieties of semigroups.

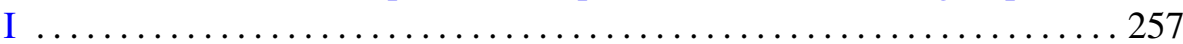

Jorge Almeida, Minimal nonpermutative pseudovarieties of semigroups.

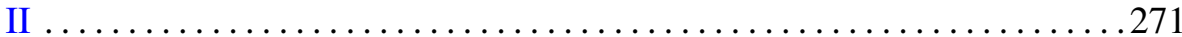

Carlos Andradas Heranz and José Manuel Gamboa Mutuberría, On

projections of real algebraic varieties $\ldots \ldots \ldots \ldots \ldots \ldots \ldots \ldots \ldots 281$

Zeev Ditzian, Inverse theorems for multidimensional Bernstein operators . . 293

M. Furi and Maria Patrizia Pera, A continuation principle for forced oscillations on differentiable manifolds $\ldots \ldots \ldots \ldots \ldots \ldots \ldots \ldots \ldots . \ldots . \ldots . \ldots 321$

James J. Hebda, The collars of a Riemannian manifold and stable

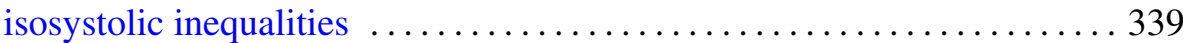

Henryk Hecht and Dragan Miličić, Character identities and asymptotic behavior of matrix coefficients of discrete series ................ 357

Piotr Jakóbczak, The boundary regularity of the solution of the $\bar{\partial}$-equation in the product of strictly pseudoconvex domains $\ldots \ldots \ldots \ldots \ldots \ldots . \ldots 371$

Krzysztof Jarosz, Isometries between injective tensor products of Banach

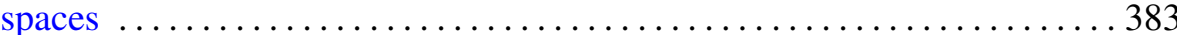

Hans Keller, On valued, complete fields and their automorphisms ........ 397

David Masser and Peter Man-Kit Shiu, On sparsely totient numbers . . . . 407

Tze-Beng Ng, Vector bundles over $(8 k+3)$-dimensional manifolds

Thomas Joseph Ransford, The spectrum of an interpolated operator and analytic multivalued functions

Akihito Uchiyama, On the radial maximal function of distributions 467 Jang-Mei Gloria Wu, On singularity of harmonic measure in space 